Original Research Paper

\title{
2D Kinematic Quantification of Soil Particles around Growing Plant Root based on Optical Mechanics
}

\author{
Wenbin Wu and Jian Zhao* \\ School of Technology, Beijing Forestry University, Beijing 100083, China
}

\author{
Article history \\ Received: 15-09-2020 \\ Revised: $16-10-2020$ \\ Accepted: 20-10-2020 \\ Corresponding Author: \\ Jian Zhao \\ School of Technology, Beijing \\ Forestry University, Beijing \\ 100083, China \\ Email: zhaojian1987@bjfu.edu.cn
}

\begin{abstract}
The quantitative kinematic description of the surrounding soil particles during root growth is a technical challenge and biologically important. In this study, a two-dimensional camera-based imaging system was used to observe micro scale interactions between plant roots and soil particles. Maize root tip was imaged during ingress into the soils. This produced a series of twodimensional images that represent temporal resolution of the geometric soil and root configurations at the micrometer scale. These images were used as inputs for full-field kinematic quantification methods, which enabled the analysis of two-dimensional deformation of the soils around elongating root. Correlationbased discrete object tracking and contour updating were used to track the shapes and the locations of soil particles and soil aggregates, while incremental digital image correlation was proposed to extract deformation and strain field within soil particle and soil aggregate. These techniques allowed the full-field displacements and strains of the soil to be quantified and the changes in shapes of soil particles to be visualized. Experimental results show that the presented shape tracking scheme, incremental digital image correlation and the research findings will be useful for the measurement and quantification of soil particle kinematics of soil-root physical interactions.
\end{abstract}

Keywords: Soil Particle, Digital Image Correlation, Kinematic Analysis, Deformation Quantification, Shape Tracking

\section{Introduction}

The root system is the dynamic interface between soil and plant. The interactions between roots and soils is particularly important for a better understanding of how plants interact with their environment (Keyes et al., 2017; 2016; Bengough et al., 2011). Roots absorb the water and mineral nutrients from the soils, assure the anchorage of the plant in soils and improve the physical properties of the soils by promoting water infiltration and gas exchange in soils (Sugai et al., 2020; Ruiz et al., 2015). On the other hand, the root trajectory depends highly on the presence of obstacles at the root scale (Kolb et al., 2017). To penetrate soil, an individual root must displace soil particles by a combination of rigid-body translation and rotation, shear and compression, which result in soil structural restoration and reorganization (Keyes et al., 2016). The quantification of the mechanical process of soil deformation around roots remains a methodological challenge. This task is difficult due to the obvious facts that direct observation and dynamic data acquisition of root and soil is obstructed by opaque soil.

To capture the behavior of soil and soil-root interactions, it is necessary to obtain deformation information from the bulk of the soil (Bull et al., 2020). Advanced experimental methods using imaging techniques (e.g., X-ray Computed Tomography (XCT) or time lapse synchrotron X-ray Computed Tomography (4D CT)) have been shown to work well for capturing three-dimensional information of soils and roots (Perret et al., 2007; Peth et al., 2010; Keyes et al., 2017). Image correlation method (e.g., Particle Image Velocimetry (PIV), Digital Image Correlation (DIC) or Digital Volume Correlation (DVC)) have been developed to measure the displacement and strain fields around a growing root, (Vollsnes et al., 2010; Peth et al., 2010; Keyes et al., 2017). These imaging techniques are expensive and not feasible for most researchers. Camera-based imaging systems are still a promising tool for investigating deformation mechanics of porous soils (Bao et al., 2018). Most of the analyses to soil deformations around growing root usually treat the soil as a granular continuum. However, the continuum assumptions break down on the rhizosphere-scale. Furthermore, the shape changes and strain field of single soil particle or soil aggregate during soil penetration by plant roots and details of the mechanical processes involved have rarely been studied and quantified. There 
is thus a need for a method to quantify the deformation and strain of soil particles at the single root scale.

In this study, the two-dimensional kinematics of soil particles around the root tips of maize seedlings has been discussed through the visualization of the changes in shape of soil particle and the quantification of the deformation field of soil particles by incremental DIC analysis with arbitrary region of interest. The remainder of present paper is organized as follows. Section 2 briefly explains the principle of incremental DIC, shape tracking scheme and experimental details. 2D kinematic quantification results of motion and deformation of the soil particles during root penetration will be presented in section 3. Section 4 provides the summary and prospect of the paper.

\section{Materials and Methods}

\section{Measurement of Soil Deformation with Incremental $D I C$}

Digital Image Correlation (DIC) is an on-contact optical full-field deformation measurement technique, which computes full field components of material deformation in the predefined Region Of Interest (ROI) on the speckled surface (Pan, 2009; Pan et al., 2010). It has been applied in the fields of pedology and phytology (Bao et al., 2018; El Hajjar et al., 2020). Subset-based DIC uses image registration algorithms to track the relative displacements of Points Of Interest (POI) between a reference image and a current deformed image. A schematic illustration of the procedure is shown in Fig. 1. In conventional DIC, all the subsequent deformed images are compared with a fixed reference image. However, for the deformation measurement of soil particles, in addition to the distinct changes in the shape and position of the soil particles, the surface texture difference between the particles is not obvious, resulting in the decor relation effect between the reference image and the deformed image. In this study, considering that the serious decor relation effect in the two images cannot be overcome by the most robust matching algorithm, incremental digital image correlation was used to deal with the decor relation effect. The procedure of the incremental DIC method used in this study is similar to that of (Pan et al., 2012). A brief principle of the method is given below. Firstly, the images of the reference and the subsequent deformed states are captured. Points of interest in the original reference image are used to find their corresponding locations in the first deformed image. In the following correlation analysis, the image recorded just before the current deformed image is used as an updated reference image and the incremental deformation vectors of each point of interest are detected. Finally, all the rest deformed images are compared with the updated reference image and the incremental displacement fields are subsequently accumulated to determine the overall displacement fields.

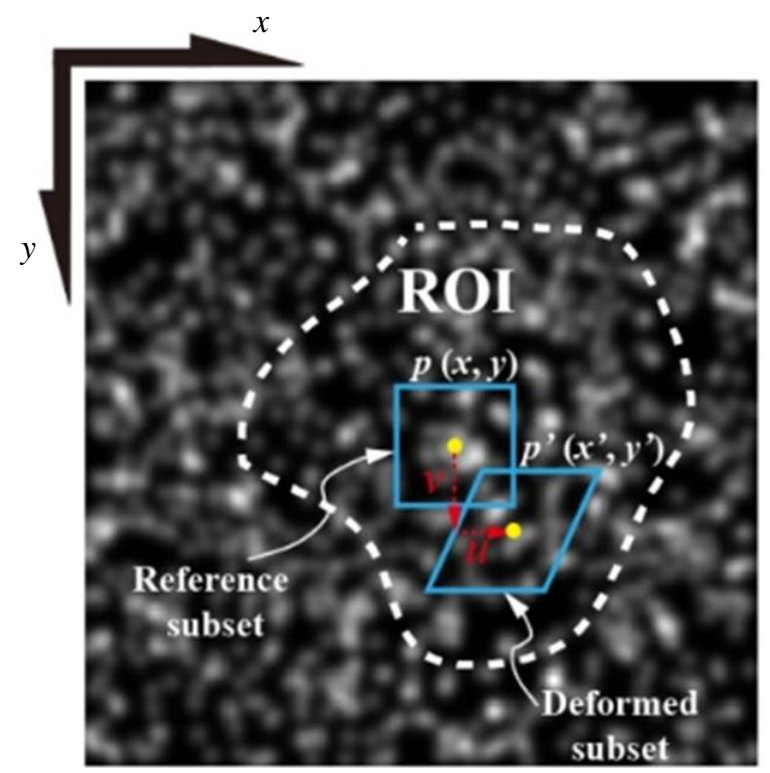

Fig. 1: Schematic illustration of the procedure of digital image correlation analysis

\section{Shape Tracking using Updating ROI}

Different from the kinematics of rigid particles, soil particles and aggregate have obvious deformation and strain during the movement. In order to track the soil particle or soil aggregate at each time step, each discrete particle or aggregate must be uniquely identified in its reference and post-deformation state. The displacement of a particle or aggregate is then defined as a vector connecting the centroid coordinates of these states. The correlation description is used to match particles or aggregates based on the incremental digital image correlation method.

Before the implementation of DIC analysis, a ROI should be defined in the reference or the current image (Blaber et al., 2015). The points within the ROI are considered as points of interest to be computed and analyzed. In the present study, we focused on the shape and displacement changes of individual soil particles or soil aggregates. Therefore, the ROI was irregular and was exactly the contour of the particles or aggregates. An important part of this process is to updating the contour of the ROI. The approach starts by forming a boundary around the ROI. The boundary is then updated according to the displacement values obtained from the last calculated displacement field of the last reference image. This is achieved by sampling the last calculated displacement field at the values at the boundary and then adding these displacements to the boundary coordinates. The boundary coordinates are treated as a polygon and the polygon filling algorithm is used to update the contour. The specific implementation steps are as follows: 
Step 1: Setting the contour of soil particle or aggregate and forming the ROI. The irregular ROI of soil particle can be obtained quickly using interactive image segmentation by maximal similarity based region merging (Ning et al., 2010).

Step 2: Updating this contour based on displacement values obtained through the last calculated displacement field for the last reference image by incremental-DIC. This happens by sampling the last calculated displacement field at the contour of ROI. These displacement values are then added to the contour coordinates.

Step 3: The contour coordinates are treated as a polygon and are used through a polygon fill algorithm to update the contour of the particles. The shape tracking process is demonstrated in Fig. 2.

\section{Strain Calculation}

Considering that the strain involves differential sensitivity to noise, which means that any noise in the displacement field will amplify the error in the strain field, the Green-Lagrangian strain is adopted and the shear strain is obtained by the following four displacement gradients:

$$
\varepsilon_{x y}=\frac{1}{2}\left(\frac{\partial u}{\partial y}+\frac{\partial v}{\partial x}+\frac{\partial u}{\partial x} \frac{\partial u}{\partial y}+\frac{\partial v}{\partial x} \frac{\partial v}{\partial y}\right)
$$

A least squares plane fit on a subset of displacement data is used to find the plane parameters in Equation 1 and the process is applied to the entire displacement field in order to obtain a shear strain field.

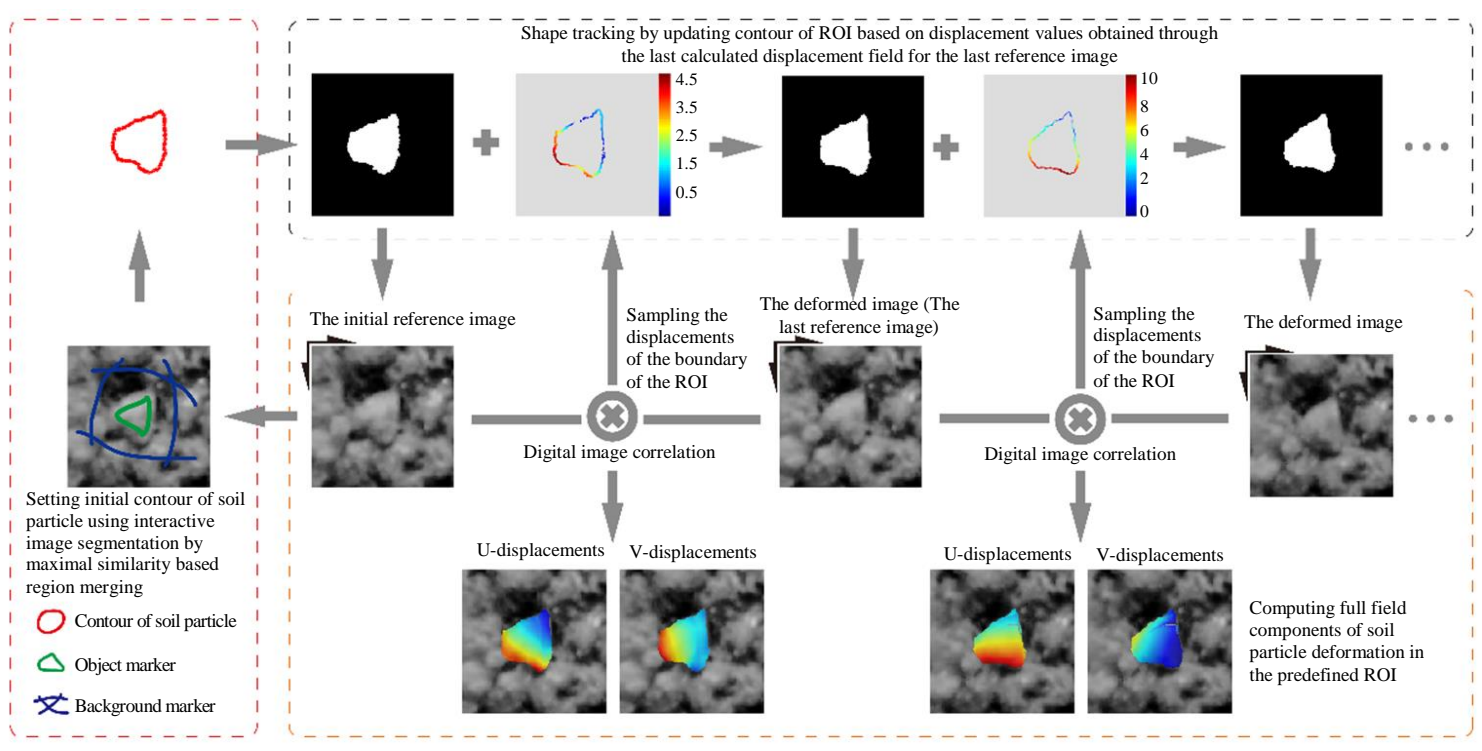

Fig. 2: Schematic illustration of the procedure of the shape tracking

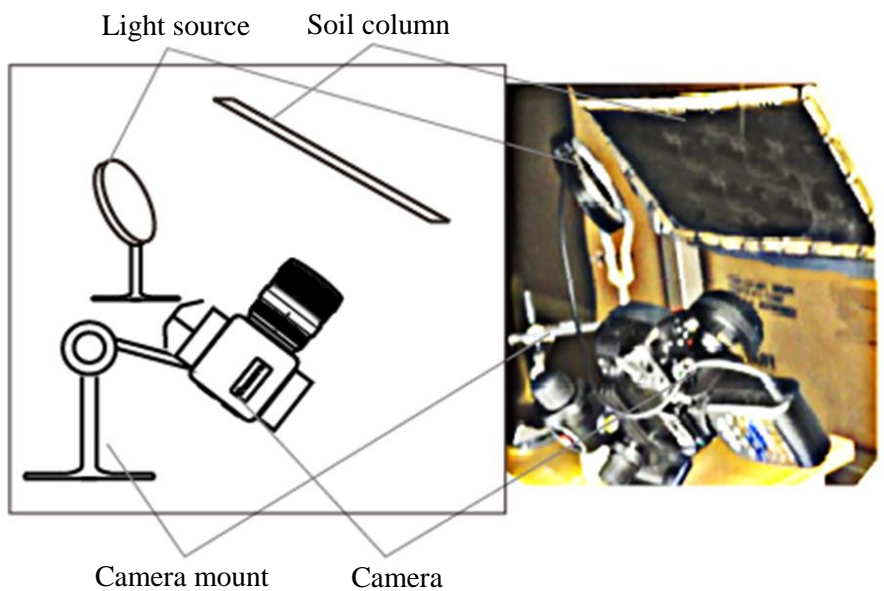

Fig. 3: Schematic and installation diagram of the experiment 


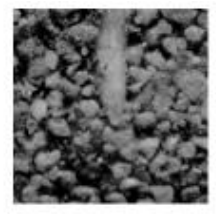

$\mathrm{t}=0$

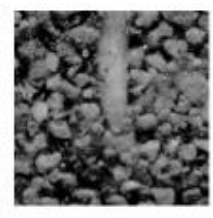

$\mathrm{t}=10 \mathrm{~min}$

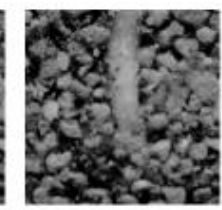

$\mathrm{t}=20 \mathrm{~min}$

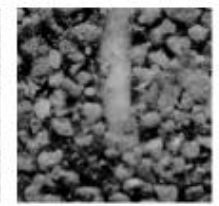

$\mathrm{t}=30 \mathrm{~min}$

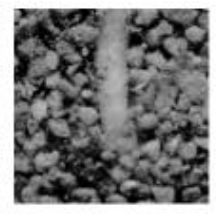

$\mathrm{t}=40 \mathrm{~min}$

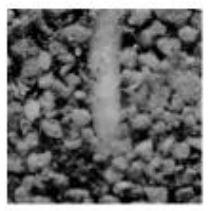

$\mathrm{t}=50 \mathrm{~min}$

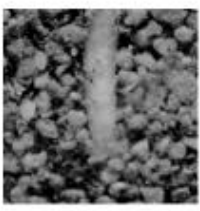

$\mathrm{t}=60 \mathrm{~min}$

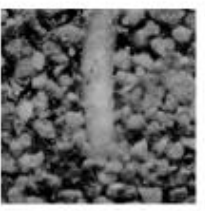

$\mathrm{t}=70 \mathrm{~min}$

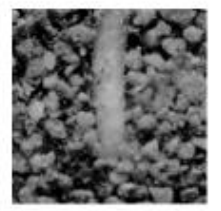

$\mathrm{t}=80 \mathrm{~min}$

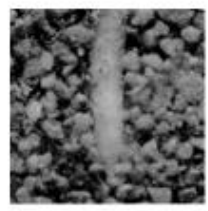

$\mathrm{t}=90 \mathrm{~min}$

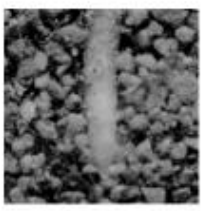

$\mathrm{t}=100 \min$

Fig. 4: Images of soil peristaltic motion during root growth

\section{Imaging}

In order to visualize soil particle displacement and strain and to develop method of proper analysis for shape tracking during soil penetration by plant roots, coarse soil (Ruiz et al., 2017) packed at a bulk density of 1500 to $1700 \mathrm{~kg} / \mathrm{m}^{3}$ was used. The schematic and installation diagrams of the experiment are shown in Fig. 3. The image acquisition system includes CCD camera, camera mount and white light source. A rectangular soil column $(20 \times 8 \times 1 \mathrm{~cm})$ was fixed at a $30^{\circ}$ incline to take advantage of gravitropism, which forces maize roots to grow along the inner surface of the column. The images of maize roots and surrounding soil particles were captured by a digital camera every 10 min during a 100 min period. Figure 4 shows the images of soil penetration by plant roots which were used to investigate the shape and position changes of the soil particles, further to reveal the two-dimensional dynamic geometrical interaction of rhizosphere soil.

In this case, the root diameter is equivalent to the soil particle size. Root penetration provokes the displacements of the neighboring soil particles which in turn modify the distribution of pores and affect the further root growth that may reorient.

\section{Results and Discussion}

\section{Qualitative Observations}

As shown in Fig. 4, discrete soil particles or aggregates were clearly distinguishable from the gasfilled pore spaces and the shapes and positions of soil particles near the root can be seen to change, that changes the microstructure. Complex particle-scale interactions between the root and the soil were observable in Fig. 5. The root passing through the soil directly changes the soil pore space and induces the soil particle displacement, which mainly occurs in the axial displacement in front of the root and the radial displacement near the root (Meurer et al., 2020).
The yellow arrow indicates root elongation during the growing period. A series of soil particles and aggregates (highlighted in red) initially located at the lateral of the initial position of the root tip are translated to form a compacted structure by large displacements that are essentially aligned to the direction of root growth. A soil aggregate (highlighted in blue) situated beneath the initial root tip position are divided into several particles that are displaced substantially parallel to the direction of root growth. A macropore (highlighted in green) situated beneath the initial root tip position allows the root to elongate with minimal soil disturbance.

\section{Kinematic Quantification}

At the particle-scale, the soil-root mechanical interactions used in this study can be described by the conceptual description shown in Fig. 6. The kinematics of each soil particle or aggregate in response to a root elongation vector $r$ (solid white arrows) is described by displacement vector $d$ (solid black arrows). $\varphi$ and $\theta$ represent the angle between the root elongation vector and the vertical direction and the angle between the displacement vector of soil particles and the root elongation vector, respectively.

\section{Root Elongation Kinematics}

Because the gray distribution of root and soil pixels overlapped, the root morphology in the captured images was segmented manually. The displacement vectors of root tip at each time-step in the process of root penetrating the soil is shown in Fig. 7a. As can be seen from the Fig. 7a, the root system remains essentially geotropic in growth, with several bends due to morphological changes resulting from penetrating the soil or growing towards macropore areas with less resistance. The quantized root growth displacement $r$ and direction angle $\varphi$ are shown in Fig. 7b. The results show that the root growth rate decreases when it penetrates or bypasses the soil particles in the process of growth, with 
the mean growth rate being $0.0142 \mathrm{~mm} / \mathrm{min}$. Although the root has the characteristic of growing in the direction with little resistance, the root growth rate decreases in the absence of soil resistance, as shown by the elongation displacement at the 100th min. Due to the resistance of soil particles, the growth direction of root oscillates vertically downward on both sides, with a mean direction angle of 10.2 degrees.
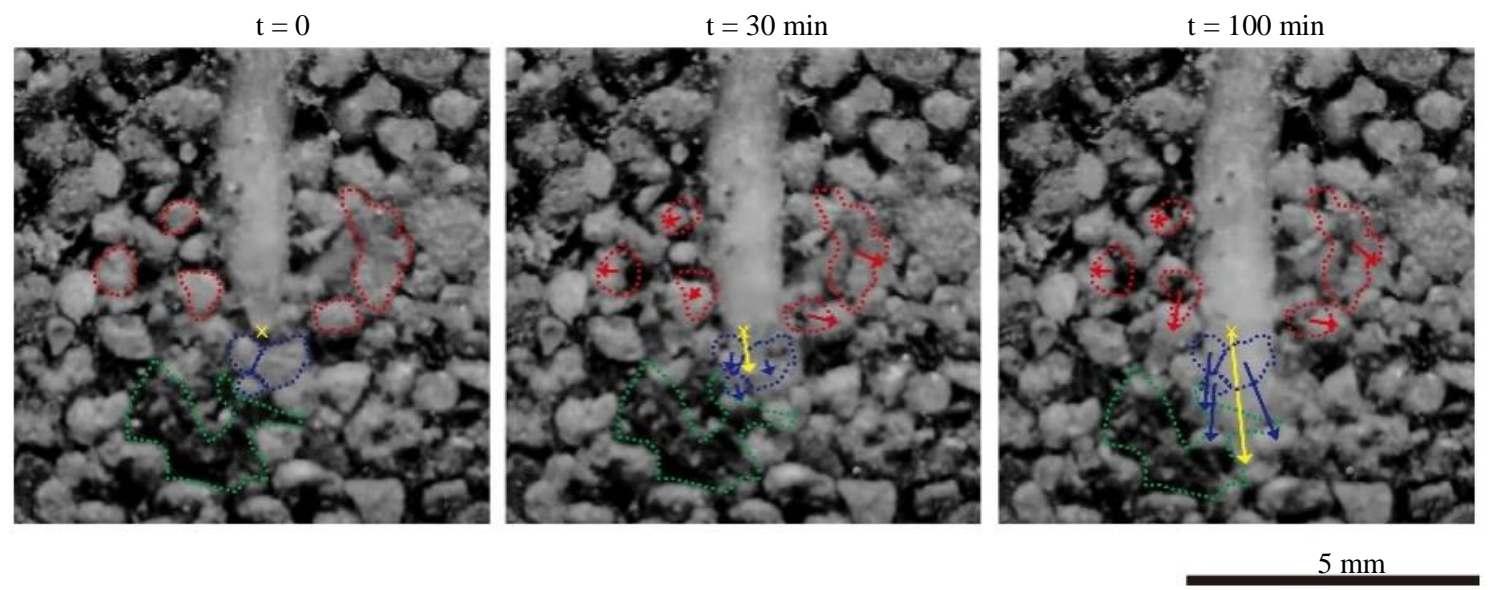

Fig. 5: Particle-scale interactions between the root and the soil

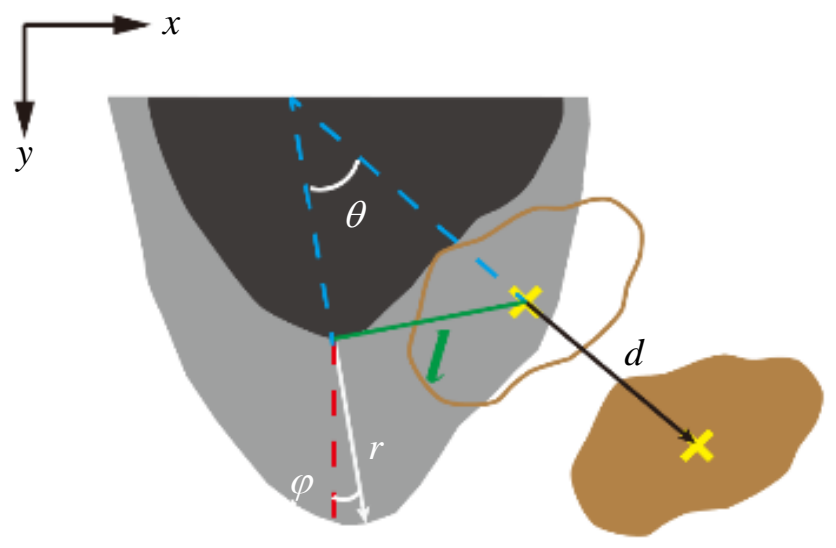

Fig. 6: Schematic of the root-soil mechanical interaction

(a)

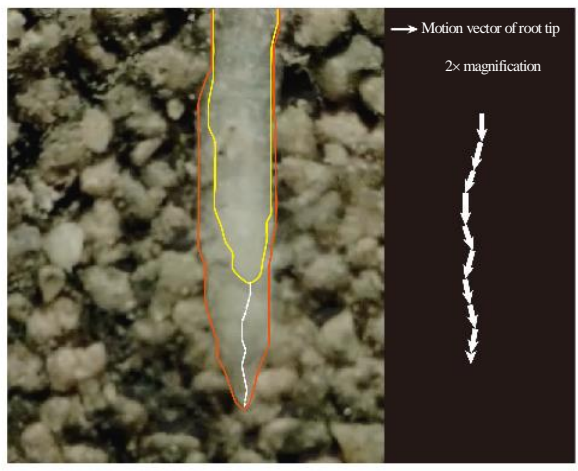

(b)

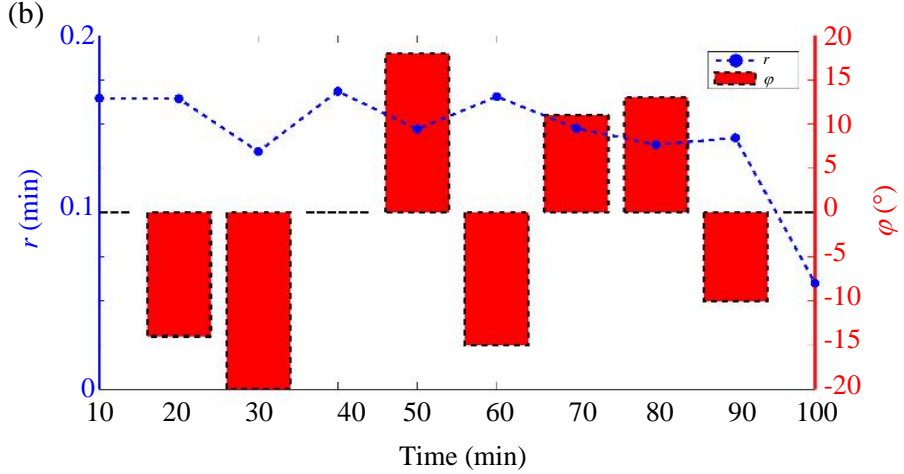

Fig. 7: Root elongation kinematics. (a) the displacement vector of the root tip (b) quantized root growth displacement $r$ and direction angle $\varphi$ 


\section{Soil Particle Kinematics}

To evaluate the shape changes of soil particles or aggregates, the shape tracking scheme based on updating ROI has been applied to the images. During the analysis of soil particle shape change using the proposed method, eight representative soil particles around the maize root were chosen, corresponding contour of each particle or aggregate, as shown in Fig. 8, was specified to be the region of interest. Figure 8 shows the changes in the shape of soil from the initial state to three intermediate states.

After tracking changes in the shape of some representative soil particles, the displacements of the particles in two dimensions are denoted by vector notations. Figure 9 shows the two-dimensional displacement vectors for some of the soil particles from the initial state to the final state. The displacements of the soil were shown to be well evaluated by the movement of the soil particles. The movement of the soil particles near the root is more obvious than that far away from the root.

In order to describe and compare the kinematics of soil particles or aggregates around growing root, three parameters were defined: Normalized displacement intensity $(\bar{d})$, angle between the displacement vector of soil particles or aggregates and the root elongation vector $(\theta)$ and normalized Euclidean distance from the root tip $(\bar{l})$.

Considering the heterogeneity of root elongation rate, displacements of soil particles or aggregates were normalized to the root elongation for the corresponding step, giving a normalized displacement intensity ( $\bar{d})$, computed according to:

$$
\bar{d}=\frac{|d|}{|r|}
$$

where, $d$ is the displacement vector of centroid of a soil particle or aggregate at the given step and $r$ is the root tip growth vector at the same step. Thus, a soil particle or aggregate which was displaced by the same magnitude as the root tip growth returned a normalized displacement intensity of $\bar{d}=1$.

The angle between the displacement vector of soil particles or aggregates and the root elongation vector is given by:

$\theta=\cos ^{-1}\left(\frac{d \cdot r}{|d| \cdot|r|}\right)$

The positive and negative signs of the angle between the displacement vector and root elongation vector are specified as follows: Take the root elongation vector as the beginning edge, the angle is counterclockwise for positive, otherwise is negative.

The normalized Euclidean distance from the root tip at a given time-step for a soil particle is expressed as:

$\bar{l}=\frac{l}{\max (l)}$

where, $l$ is the Euclidean distance from the root tip to the centroid of the soil particle at a given time-step and $\max (l)$ represents the maximum value of $l$ for the all time-steps.
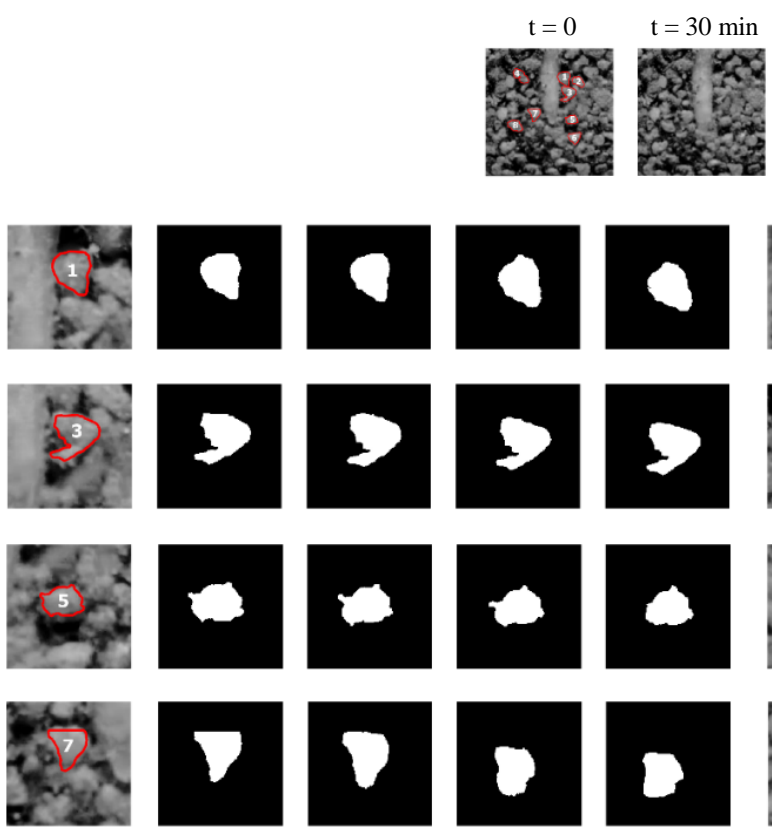
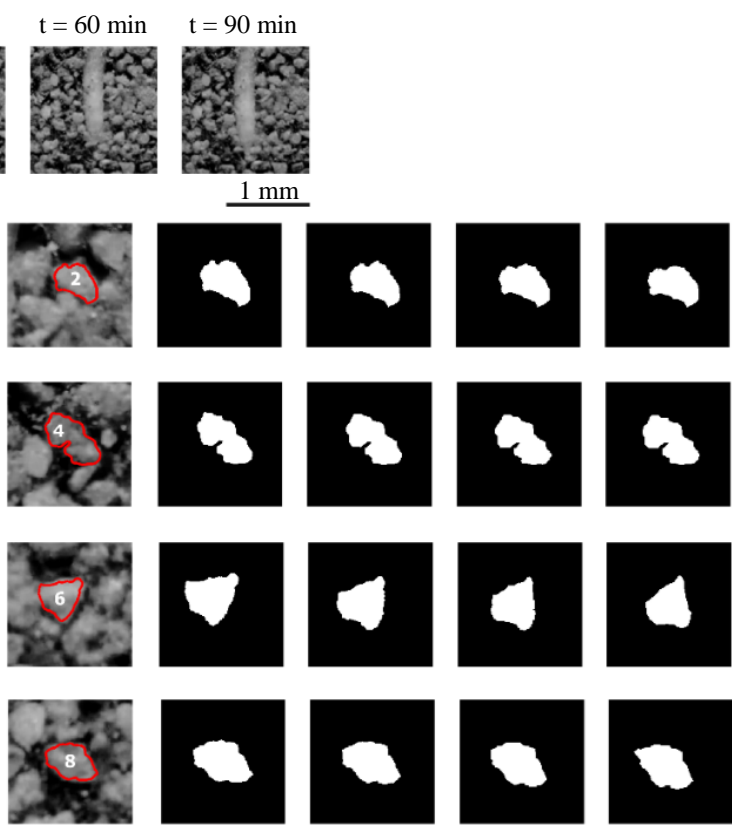

$\underline{0.2 \mathrm{~mm}}$

Fig. 8: The changes in the shape of representative soil particles from the initial state to three intermediate states 

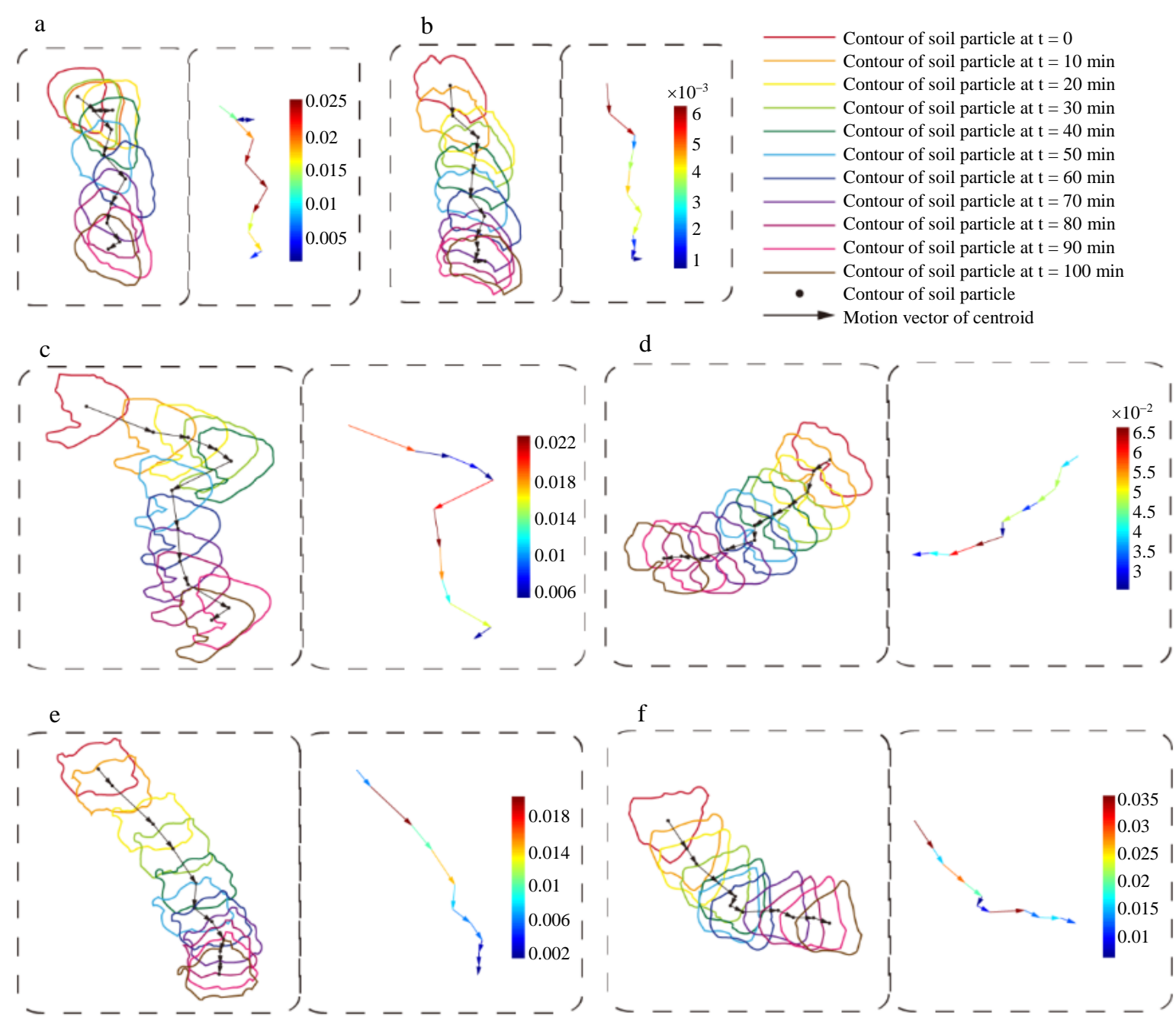

$\mathrm{f}$

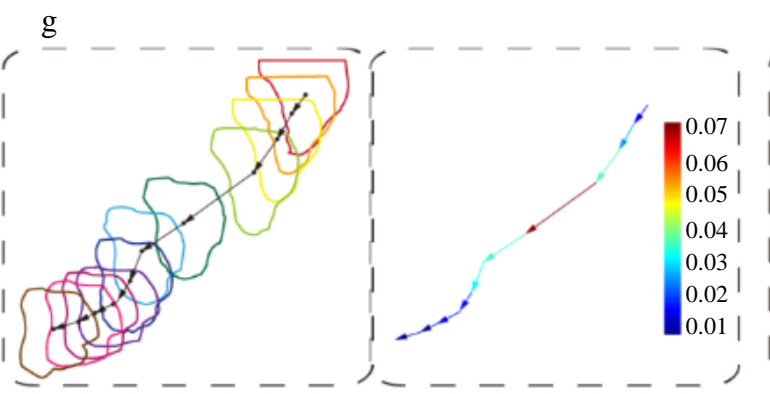

$\mathrm{h}$
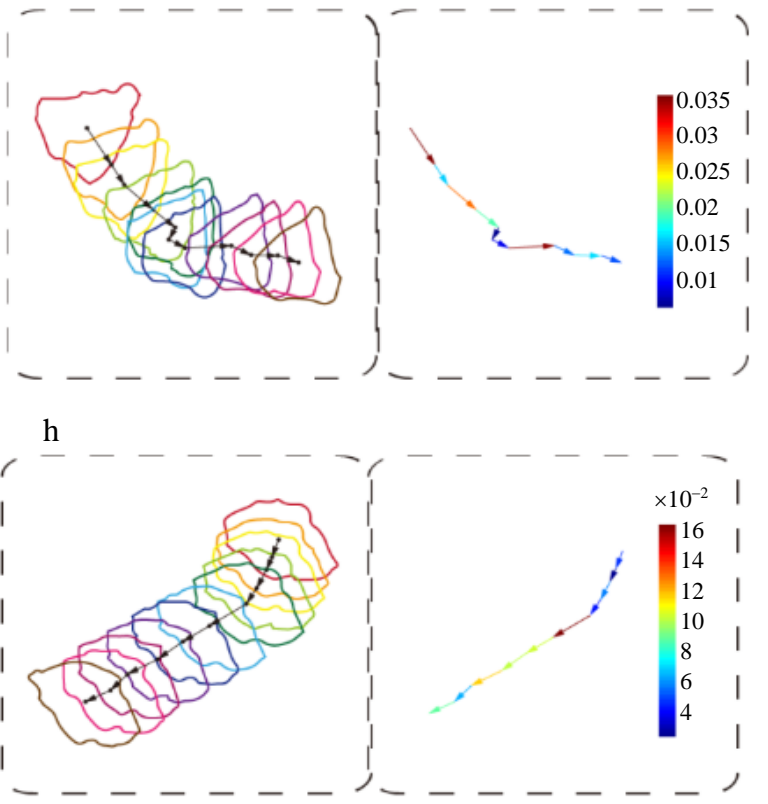

Fig. 9: Two-dimensional displacement vectors for some of the soil particles from the initial state to the final state

Figure 10 shows the above three kinematic parameters of the eight representative soil particles, plotted with respect to time-step. For soil particles with the initial position above the root tip (e.g., 1-5,7), the normalized Euclidean distance $(\bar{l})$ from the root tip increased with the increase of time step, while for soil particles with the initial position below the root tip (e.g., 6 , 8), the normalized Euclidean distance $(\bar{l})$ first decreased and then increased with the increase of time step. The normalized displacement intensity $(\bar{d})$ and the angle between the displacement vector of soil particle and the root elongation vector $(\theta)$ are affected by the root tip displacement and the distance to the root. The normalized displacement intensity and the angle between the displacement vector and root elongation vector of the soil particles in direct contact with the root change more dramatically (e.g., 1 and 3), which also occurs when soil particles interact with each other (e.g., 2 and 1, 3). The 
normalized displacement intensity of soil particles close to the root and surrounded by macroscopic pores fluctuates greatly (e.g., 5,7), because the presence of macroscopic pores allows enough space for the soil particles to move and adjust. Regardless of the initial location above or below the root tip, the normalized displacement intensity of soil particles far away from the root changes gently (e.g., 4,6,8) and the angle between the displacement vector and root elongation vector basically keeps the same direction, moving in the direction perpendicular to the root to compacted the soil.

\section{Soil Particle Strain}

Using the incremental DIC method and updating ROI, the calculated horizontal and vertical displacement field so fall eight soil particles are shown in Fig. 11. From the displacement maps, we can draw the conclusion that the decor relation effect caused by small texture difference is overcome by incremental DIC method. Based on the displacement fields shown in Fig. 11, the full-field shear strain distributions of each soil particle can also be estimated. The results in Fig. 11 further indicate that the root directly changes the soil pore space by passing through the soil and induces soil particle displacement, which occurs predominantly axially in front of the root and radially beside the root. With the elongation of the root, the diameter of the root gradually increases and the soil on both sides of the root appears local deformation under the compression of the root, which is inversely proportional to the distance to the root: The further away from the root, the smaller the deformation of the soil particles. The existence of voids around soil particles makes the soil have more movement space, so it will also produce a large displacement, which is basically uniform deformation. When soil particles are in close contact with the surrounding soil or roots, uneven deformation will occur due to uneven forces.

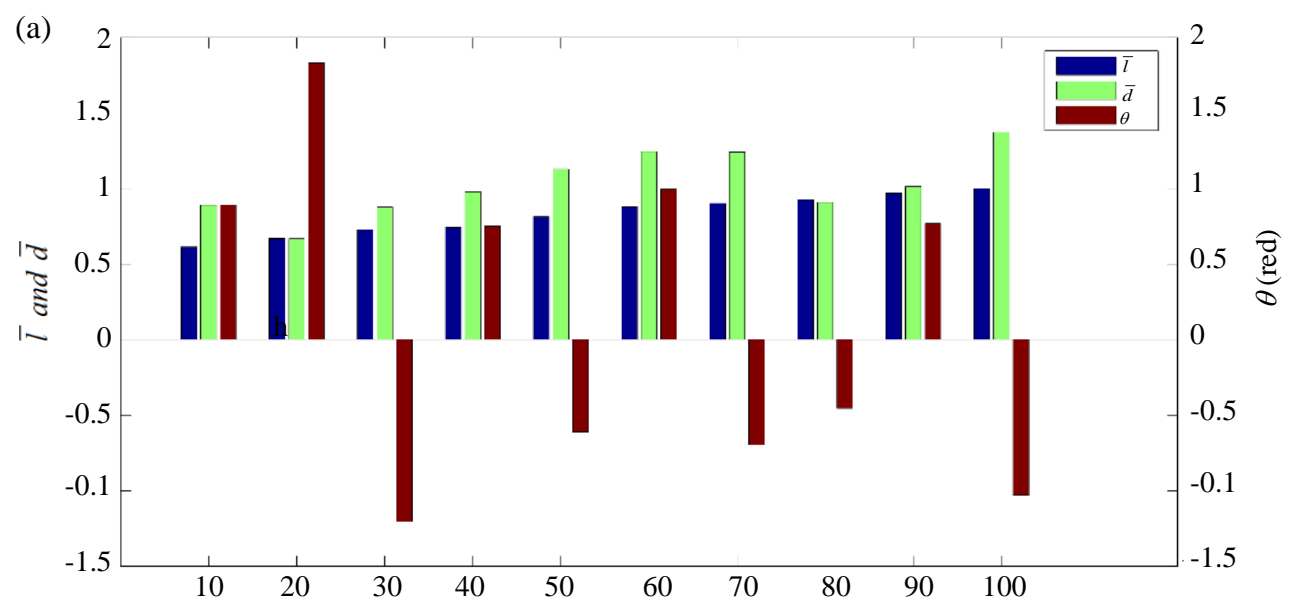

(b)

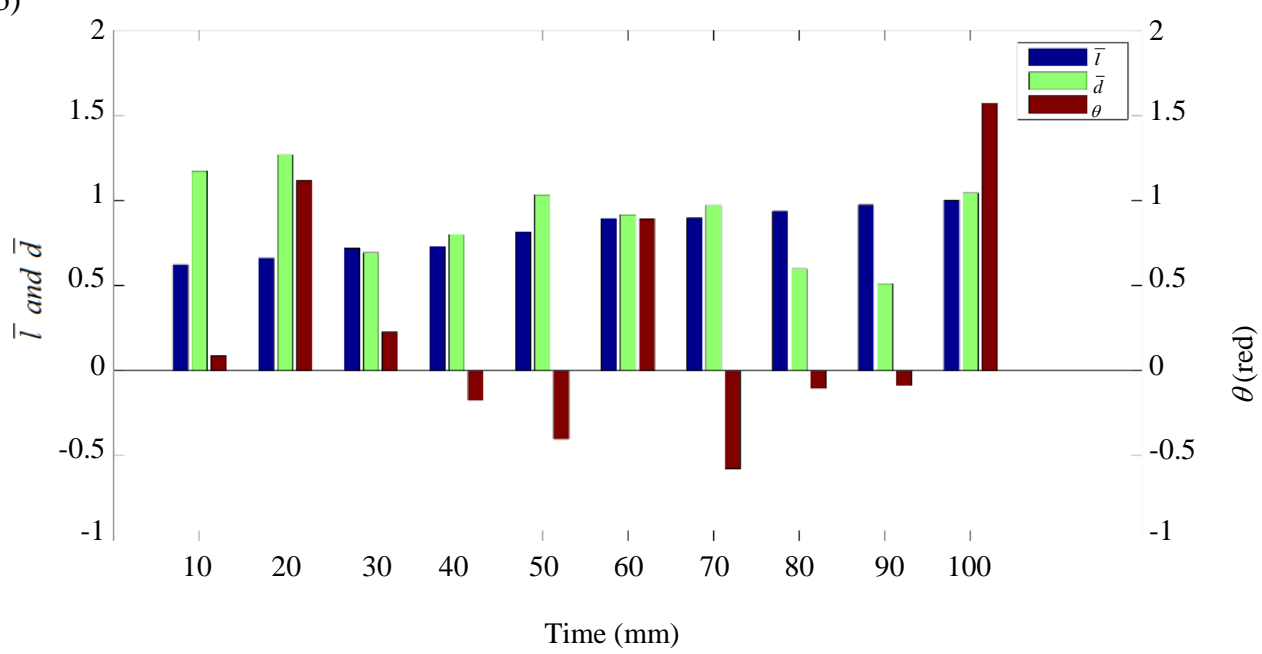


Wenbin Wu and Jian Zhao / American Journal of Biochemistry and Biotechnology 2020, 16 (4): 494.506

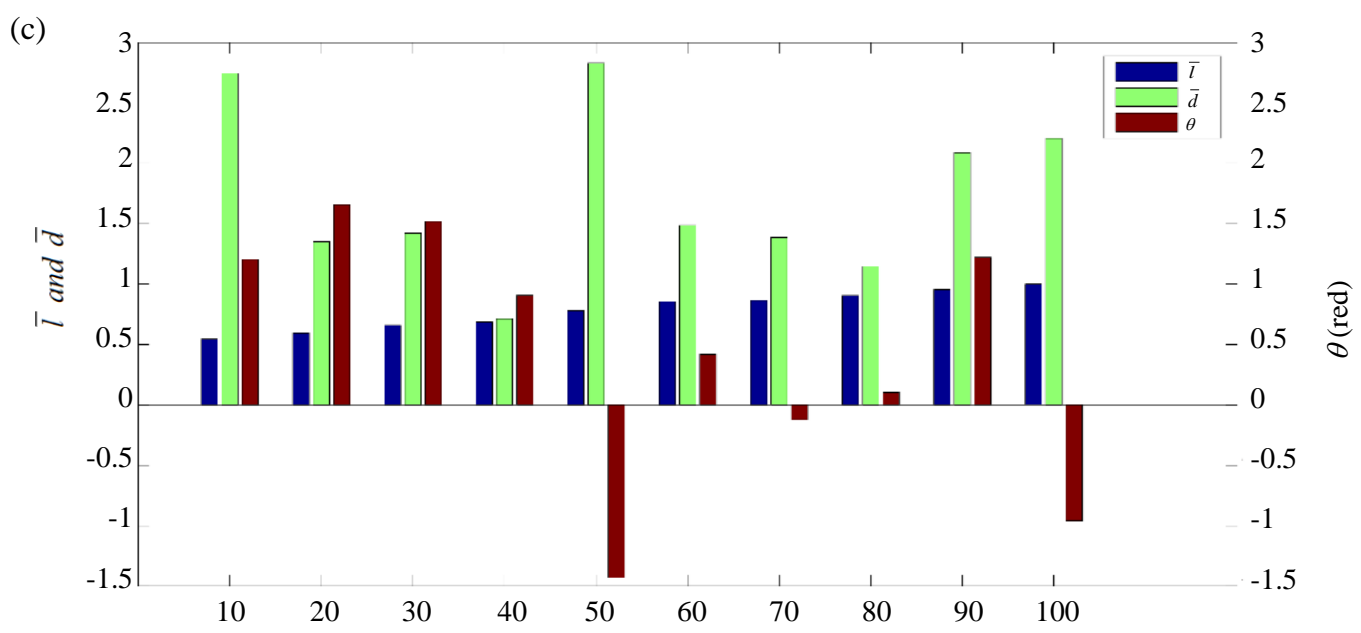

Time (mm)

(d)

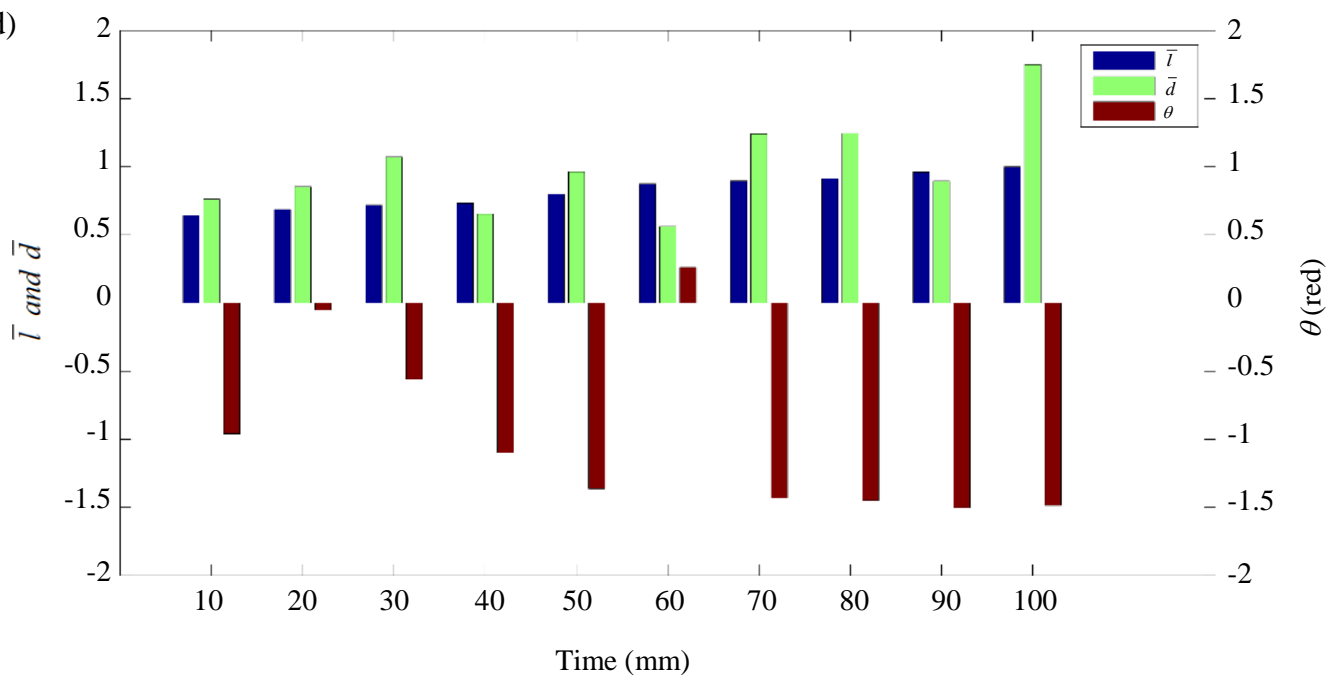

(e)

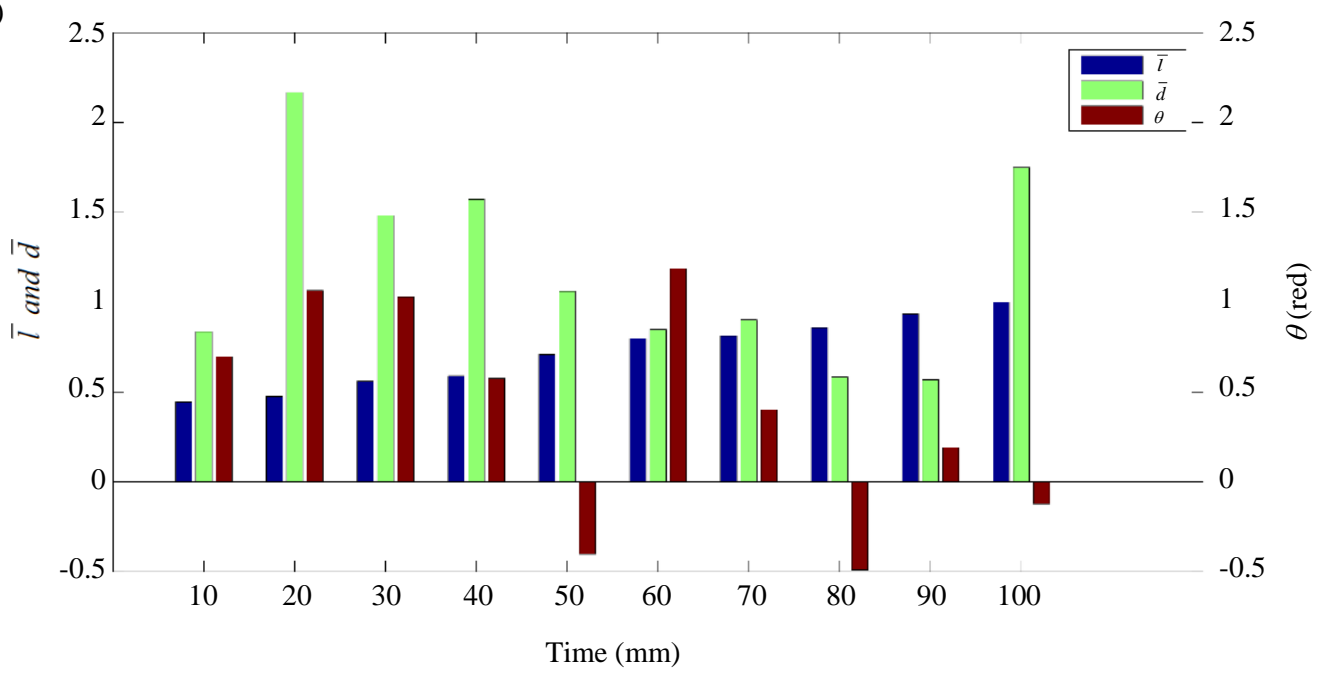


(f)

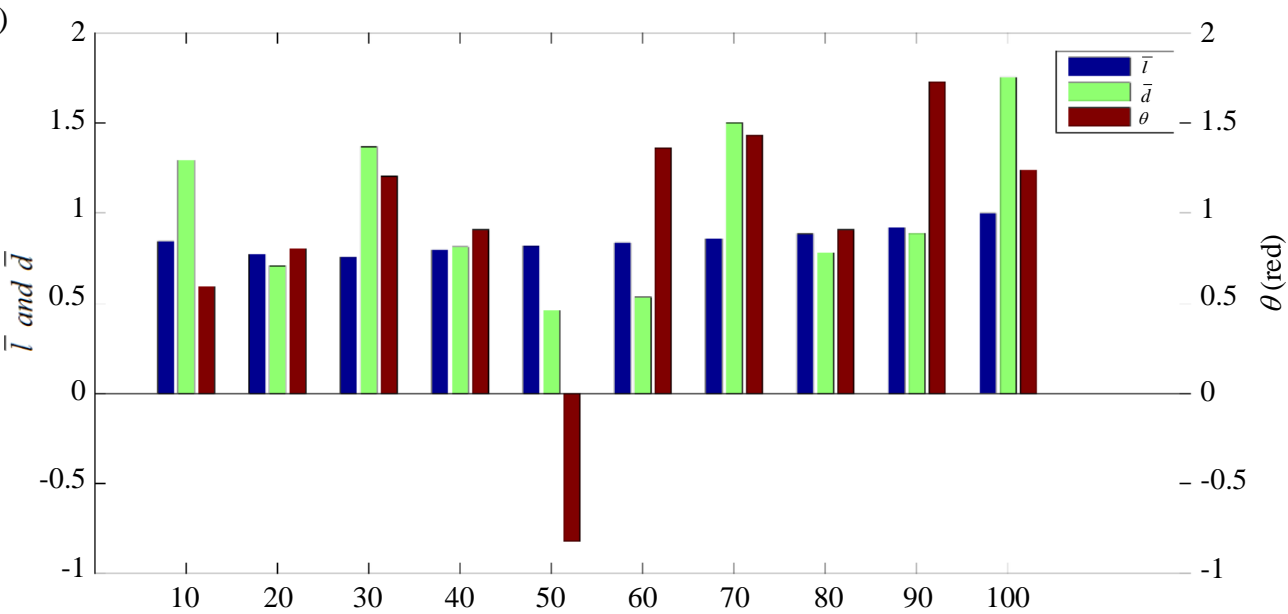

Time (mm)

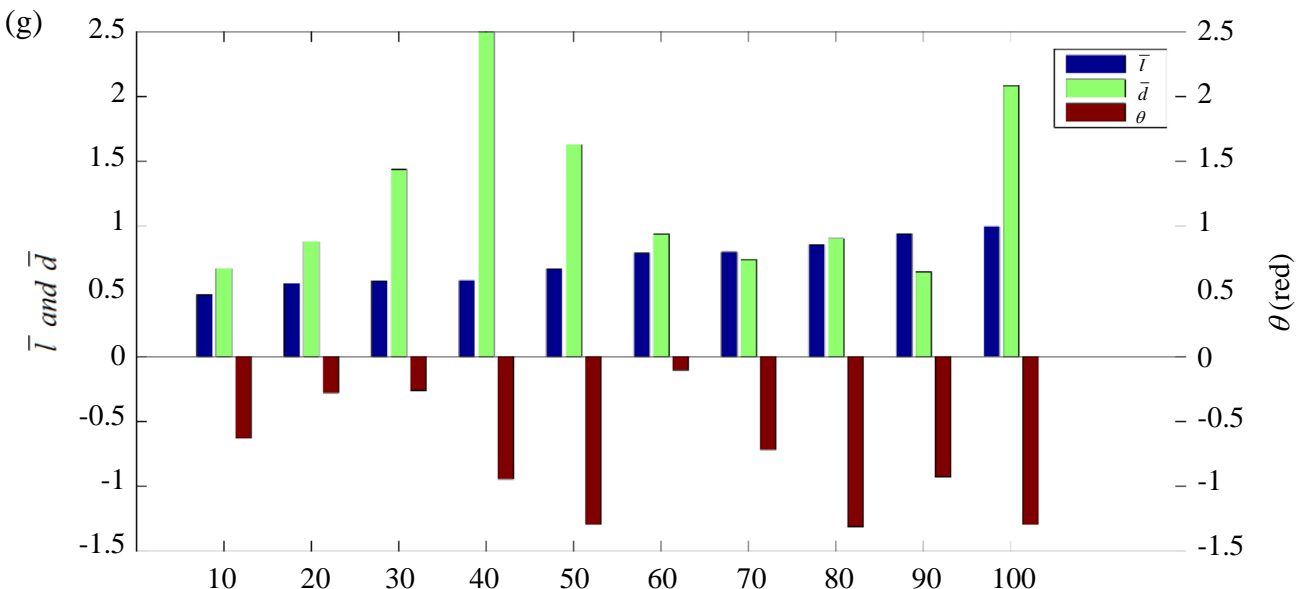

Time $(\mathrm{mm})$

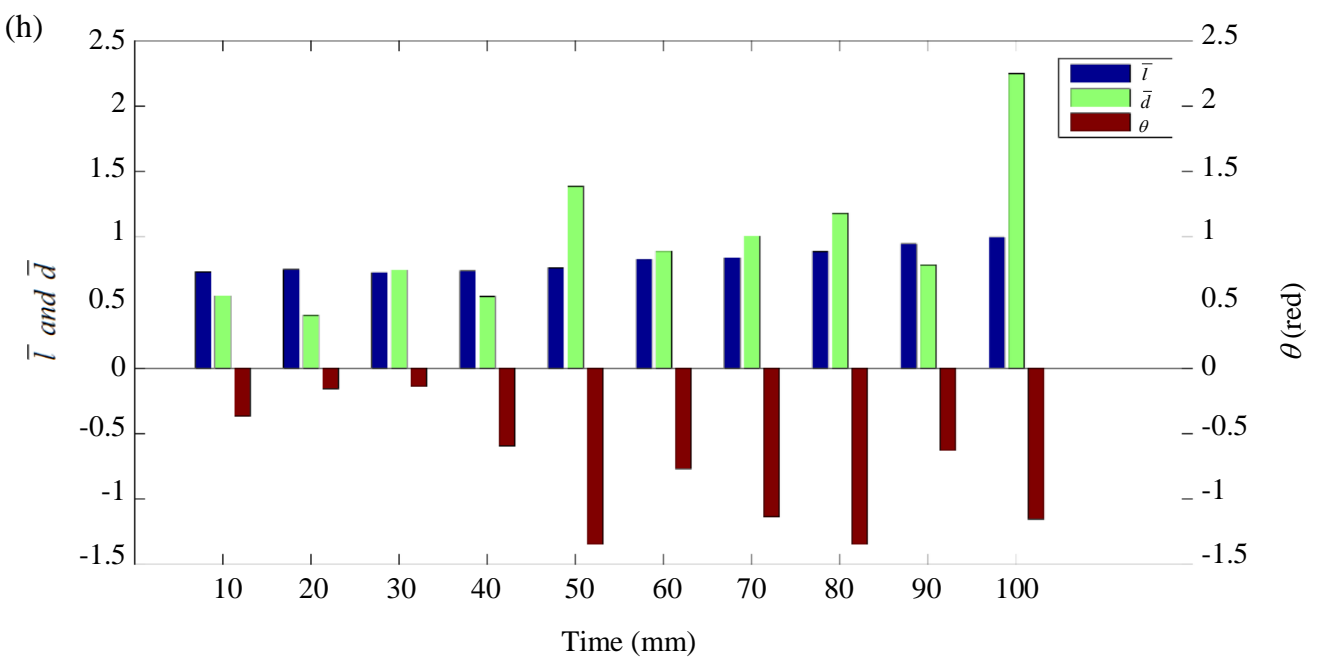

Fig. 10: Quantitative results of three kinematic parameters for the eight representative soil particles 

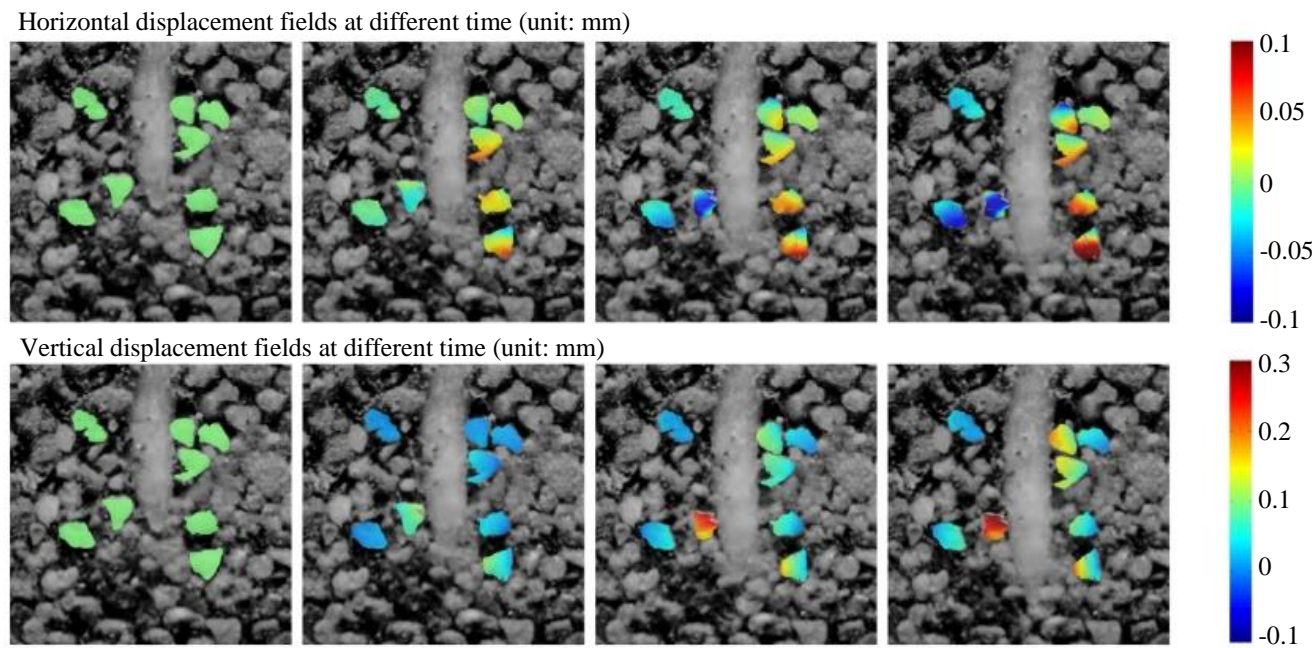

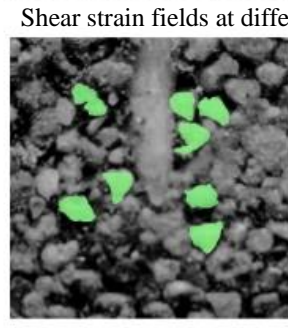

$\mathrm{t}=0$

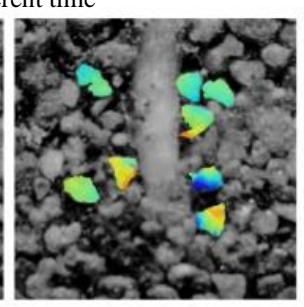

$\mathrm{t}=30 \mathrm{~min}$

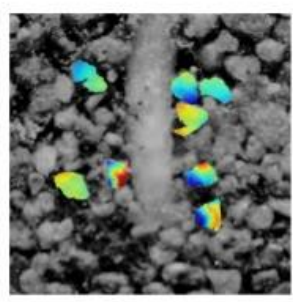

$\mathrm{t}=60 \mathrm{~min}$

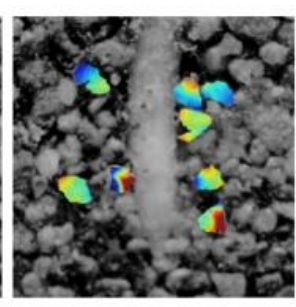

$\mathrm{t}=90 \mathrm{~min}$

Fig. 11: Calculated horizontal, vertical displacement and shear strain fields of all eight representative soil particles using incremental DIC analysis

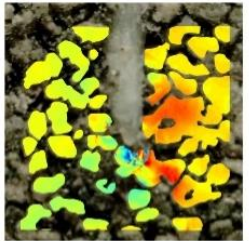

(a)

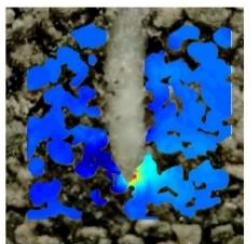

(f)

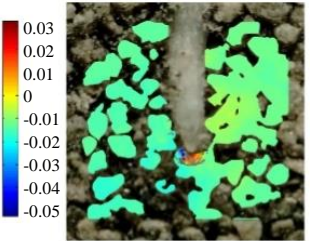

(b)

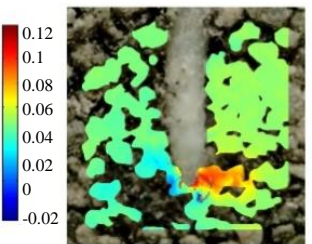

(g)

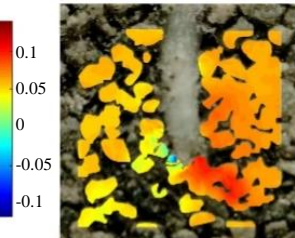

(c)

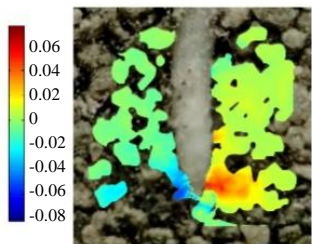

(h)

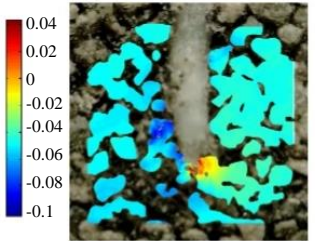

(d)

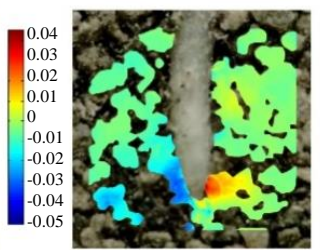

(i)

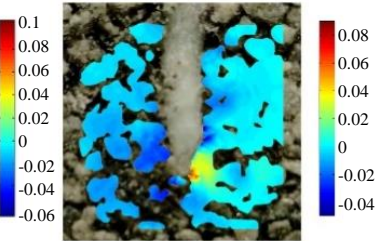

(e)

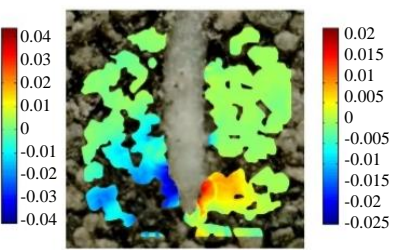

(j)

Fig. 12: Full-field shear strain shown for each time step

Based on the previous results, the calculated shear strain fields of the soil particles and aggregates are shown in Fig. 12. It can be seen from the figure that the shear strain of the soil far away from the root tip is small and uniform and the soil particles and aggregates that are passed through or bypassed by the root tip near the root tip produce relatively large shear strain. The DIC measurements provide full-field displacement and strain measurement for soil particles and aggregates, allowing for the visualization and analysis of strain field variations that occur due to the soil-root interaction (Wang et al., 2020). 2D-DIC is an effective measurement tool for planar soil particles and aggregates that do not exhibit out-of-plane displacement or strain. The displacement of soil particle and the strain within each particle and aggregate were detected using the DIC 
measurement, indicating sufficient particle contrast. The strain data in Fig. 12 show that high strain areas of the soil appear near root tips, revealing a correspondence with visible roots, providing evidence that the measured strain is due to root growth rather than other phenomena. The strain generated when the root penetrates the soil particles is significantly larger than that generated when the root passes around the soil particles. Combined with the growth of root over time as shown in Fig. 7, this method can not only obtain static data of root and soil, but also capture dynamic phenomena.

\section{Conclusion}

In this study, the shape, displacement, shear strain of individual soil particle around plant root, as well as the kinematics of soil particle were measured by incremental DIC and shape tracking scheme. The main conclusions drawn from this paper are listed as follows: (1) In order to determine the mechanical properties of soil, quantitative information about the displacement and strain field is required. In this study, the incremental DIC method provided a reliable quantification of the displacements and shear strains of individual particles through which the kinematic behaviors of soil particles were clearly represented. (2) Through direct macroscopic observation, changes in the shape and position of soil particles were clearly observed. The proposed shape tracking scheme, applied along with the incremental DIC to the soil images in the present study, has successfully tracked the shape of representative soil particles around the roots during root penetration.

It should be noted that, due to the limitations of the two-dimensional imaging system itself, only planar images can be collected and the three-dimensional aspects of soil and root system are ignored. The effectiveness and flexibility of DIC based visualization analysis method for root soil interaction are expected to promote more creativity in root and soil research, especially in the field of dynamic response of root-soil interaction.

\section{Acknowledgement}

This work was supported by Fundamental Research Fund for the Central Universities (No. 2018ZY08).

\section{Author Contributions}

Wenbin Wu: Wrote the original draft and analyzed the data.

Jian Zhao: Provided the theoretical support and improved the manuscript.

\section{Conflicts of Interest}

The authors declare that there is no conflict of interest.

\section{References}

Bao, T., Melenka, G. W., Ljubotina, M. K., Carey, J. P., \& Cahill, J. F. (2018). A new method for the rapid characterization of root growth and distribution using digital image correlation. New Phytologist, 218(2), 835-846.

Bengough, A. G., McKenzie, B. M., Hallett, P. D., \& Valentine, T. A. (2011). Root elongation, water stress and mechanical impedance: A review of limiting stresses and beneficial root tip traits. Journal of Experimental Botany, 62(1), 59-68.

Blaber, J., Adair, B., \& Antoniou, A. (2015). Ncorr: Open-Source 2D Digital Image Correlation Matlab Software. Experimental Mechanics, 55(6), 1105-1122.

Bull, D. J., Smethurst J.A.,Sinclair I., Pierron F., Roose T., Powrie W., \& Bengough A. G. (2020). Mechanisms of root reinforcement in soils: an experimental methodology using four-dimensional X-ray computed tomography and digital volume correlation. Proceedings of the Royal Society A, 476, 2019. 0838.

El Hajjar, A., Ouahbi T., Eid J., Hattab M. \&Taibi S. (2020). Shrinkage cracking of unsaturated fine soils: New experimental device and measurement techniques. Strain, e12352.

Keyes, S. D., Gillard, F., Soper, N., Mavrogordato, M. N., Sinclair, I., \& Roose, T. (2016). Mapping soil deformation around plant roots using in vivo 4D X-ray Computed Tomography and Digital Volume Correlation. Journal of Biomechanics, 49(9), 1802-1811.

Keyes, S.D., Cooper, L., Duncan, S., Koebernick, N., Mckay Fletcher, D.M., Scotson, C.P., van Veelen A., Sinclair I., Roose T. (2017). Measurement of micro-scale soil deformation around roots using four-dimensional synchrotron tomography and image correlation. J. R. Soc. Interface. 14, 20170560.

Kolb, E., Legué, V., \& Bogeat-Triboulot, M. B. (2017). Physical root-soil interactions. Physical biology, 14(6), 065004.

Ning, J., Zhang, L., Zhang, D., \& Wu, C. (2010). Interactive image segmentation by maximal similarity based region merging. Pattern Recognition, 43(2), 445-456.

Meurer, K., Barron J., Chenu C., Coucheney E., Fielding M., Hallett P., \& Herrmann A. M. (2020). Aframework for modelling soil structure dynamics induced bybiological activity. Global Change Biology, 26, 5382-5403.

Pan, B. (2009). Reliability-guided digital image correlation for image deformation measurement. Applied Optics, 48(8), 1535. 
Pan, B., Dafang, W., \& Yong, X. (2012). Incremental calculation for large deformation measurement using reliability-guided digital image correlation. Optics and Lasers in Engineering, 50(4), 586-592.

Pan, B., Wang, Z., \& Lu, Z. (2010). Genuine full-field deformation measurement of an object with complex shape using reliability-guided digital image correlation. Optics Express, 18(2), 1011.

Perret, J. S., Al-Belushi, M. E., \& Deadman, M. (2007). Non-destructive visualization and quantification of roots using computed tomography. Soil Biology and Biochemistry, 39(2), 391-399.

Peth, S., Nellesen, J., Fischer, G., \& Horn, R. (2010). Non-invasive 3D analysis of local soil deformation under mechanical and hydraulic stresses by $\mu \mathrm{CT}$ and digital image correlation. Soil and Tillage Research, 111(1), 3-18.

Ruiz, S., Or, D., \& Schymanski, S. J. (2015). Soil Penetration by Earthworms and Plant RootsMechanical Energetics of Bioturbation of Compacted Soils. PLOS ONE, 10(6), e0128914.
Ruiz, S., Schymanski, S. J., \& Or, D. (2017). Mechanics and Energetics of Soil Penetration by Earthworms and Plant Roots: Higher Rates Cost More. Vadose Zone Journal, 16(8), 0.

Sugai, T., Yokoyama, S., Tamai, Y., Mori, H., Marchi, E., Watanabe, T., Satoh, F., Koike, T. (2020). Evaluating Soil-Root Interaction of Hybrid Larch Seedlings Planted under Soil Compaction and Nitrogen Loading. Forests, 11, 947.

Vollsnes, A. V., Futsaether, C. M., \& Bengough, A. G. (2010). Quantifying rhizosphere particle movement around mutant maize roots using time-lapse imaging and particle image velocimetry. European Journal of Soil Science, 61(6), 926-939.

Wang, P., Guo X., Sang Y., Shao L., Yin Z. \& Wang Y. (2020). Measurement of local and volumetric deformation in geotechnical triaxial testing using 3Ddigital image correlation and a subpixel edge detection algorithm. Acta Geotechnica, 15, 2891-2904. 